\title{
Prosthetic sockets stabilized by alternating areas of tissue compression and release
}

\author{
Randall D. Alley, CP, LP; ${ }^{*}$ T. Walley Williams III, MA; ${ }^{2}$ Matthew J. Albuquerque, CPO; ${ }^{3}$ David E. Altobelli, MD $^{4}$ \\ ${ }^{1}$ biodesigns Inc, Thousand Oaks, CA; ${ }^{2}$ Liberating Technologies Inc, Holliston, MA; ${ }^{3}$ Next Step Orthotics \& Prosthetics \\ Inc, Manchester, $\mathrm{NH} ;{ }^{4}$ DEKA Research and Development Corp, Manchester, $\mathrm{NH}$
}

\begin{abstract}
A prosthetist makes a conventional socket by wrapping plaster bandage around the residual limb and using the resulting shell to create a positive model. After he or she modifies the plaster, it is used to create a laminated socket. Such sockets are almost perfect cylinders that encapsulate the limb. The bone is centered in soft, compressible tissue that must move aside before the bone can push against the socket to transmit force or torque to the prosthesis. In a compression/release stabilized (CRS) socket, three or more longitudinal depressions compress and displace tissue between the socket wall and the bone to reduce lost motion when the bone is moved with respect to the socket. Release areas between depressions are opened to accommodate displaced tissue. Without these openings provided, the CRS socket will not function as intended. Often, the release areas of compression are the struts of a carbon-fiber frame, and the regions between struts are left open. A frame with openings may be modified by the prosthetist adding a thin membrane fully surrounding the limb but allowing the membrane and underlying tissue to enter the release openings. The membrane may contain electrodes, and it may constitute a roll-on liner that helps suspend the prosthesis. We introduce three socket designs: transradial, transfemoral, and transhumeral.
\end{abstract}

Key words: amputation, frame socket, load transmission, prosthetic interface, prosthetic socket, tissue compression, tissue release, transfemoral socket, transhumeral socket, transradial socket.

\section{INTRODUCTION}

Traditional upper-limb prosthetic sockets share certain problems. Most sockets simply contain the tissue of the remaining limb. Since a prosthetist produces them by slightly modifying casts taken by wrapping plaster bandages around the limb, the sockets are usually circular in cross section and thus encapsulate the limb. The advent of myoelectric control led to new socket designs. Transradial (TR) sockets were needed that would stabilize the location of the electrodes, and the Muenster and Northwestern sockets were introduced [1-3]. These sockets are selfsuspending but nonetheless still display a number of problems. They do not permit the user to fully flex or extend the elbow, they do not prevent lost motion between the bones of the remaining limb and the distal prosthetic structure during active lifting, and they do not load the bone uniformly but rather concentrate the load near the ends.

\footnotetext{
Abbreviations: ACCI = anatomically contoured and controlled interface, CRS = compression/release stabilized, DARPA = Defense Advanced Research Projects Agency, ROM = range of motion, $\mathrm{TF}=$ transfemoral, $\mathrm{TH}=$ transhumeral, $\mathrm{TR}=$ transradial. *Address all correspondence to Randall D. Alley, CP, LP; biodesigns Inc, 1405 Corte de Primavera, Thousand Oaks, CA 91360; 310-291-4543; fax: 805-552-0705.

Email: ralley@biodesigns.com

DOI:10.1682/JRRD.2009.12.0197
} 
Myoelectric control also changed transhumeral (TH) sockets with the introduction of the Dynamic Socket [46]. It has a low lateral trim line to prevent the lifting of the electrodes during the extremes of flexion and abduction. It also has anterior and posterior wings that stabilize the prosthesis against rotation around the long axis. Similarly, the X-frame socket [7-9] has replaced the full contact socket for amputations at the shoulder level, because it permits the user to bend forward and to move the shoulder while maintaining good contact with electrodes. It also stabilizes the prosthesis against rotation at its superior and inferior borders and covers far less surface area of the thorax for increased heat dissipation. In this article, we review the evolution of these designs with additional references by Lake [7].

\section{LONGITUDINAL DEPRESSIONS AND RELEASE AREAS DEFINE COMPRESSION/RELEASE STABILIZED SOCKET}

This article will introduce improved sockets for persons with TR, TH, and transfemoral (TF) amputations created with longitudinal depressions added in the socket walls with open release areas between the depressions that receive the displaced tissue. When the depressions and release areas are correctly located, they reduce motion of the underlying bony structures with respect to both the socket and the rest of the prosthesis. One can define the depressions and releases during cast-taking but only by radically changing the way casts are taken.

Traditionally, the prosthetist uses a plaster wrap to define the shape of the remaining limb. The typical plaster wrap results in a shell that is almost circular in cross section throughout most of its length. When the shell is filled with plaster, the prosthetist modifies the resulting positive model before creating a socket over it by laminating or by thermoforming plastic. The prosthetist then adds extra plaster to the model to create space in the socket to accommodate bony prominences and removes the plaster to tighten up the fit. The experienced prosthetist can speed up the rectification process by contouring the original cast while it is setting.

Creating a compression/release stabilized (CRS) socket requires one to apply selective pressure during cast-taking, but this pressure must be applied in a specific way. A definition of terms will help the reader to follow the discussion. We only briefly summarize the casting process here, because prosthetists must be fully trained and certified in the application of this design such that patients are not harmed because of an incomplete understanding of the process.

If during the cast-taking, the technician pushes inward toward the bone, he or she will create a depression in the resulting cast. When the depressed area is parallel to the length of the underlying bone, it will appear as a channel or longitudinal depression. Further use of the word depression in this article will describe any shape created by pushing inward and use of longitudinal depression will describe long depressions parallel to the bones underneath. If one pushes a substantial area inward while holding the limb of the amputee, this action will displace tissue in other areas outward to form bulges. When the cast is taken, the stretched plaster wrap over these bulges still applies some inward force. For a CRS socket to perform correctly, these areas should have little or no inward force where the tissues bulge. After all remaining force is removed between the longitudinal depressions, the areas between are called release areas. After we discuss the physics underlying the operation of a CRS socket in this article, we will briefly illustrate how each of the three socket designs (TH, TF, and TR) can be created using the plaster cast technique. The unique features of these sockets are the longitudinal depressions and the release areas. The release areas are critical to the functioning of this new socket design.

\section{THEORY BEHIND COMPRESSION/RELEASE STABILIZED SOCKET}

\section{Physics of Tissue Compression in Socket}

Consider a disk pushed against the skin of the forearm as shown in Figure 1. Initially the disk will push aside some of the underlying flesh. This action requires little force but yields significant motion. As the disk moves further in, the connective tissue in the fat and muscle begins to restrain tissue from further displacing sideways and reducing motion. Finally, the muscle and fat are fully compressed, and no further motion will occur. Figure 2 shows such a disk displaced versus the force required. Note that the plot begins as a straight line (area I). Then a gradual change in the slope occurs (area II). Finally, the tissue is fully compressed (area III) and the slope becomes quite steep. Forces below the transition from areas II to III can be tolerated all day without discomfort; yet only a little motion beyond this bend in the curve is possible. This anatomical fact is the key element of a CRS socket. This brief 


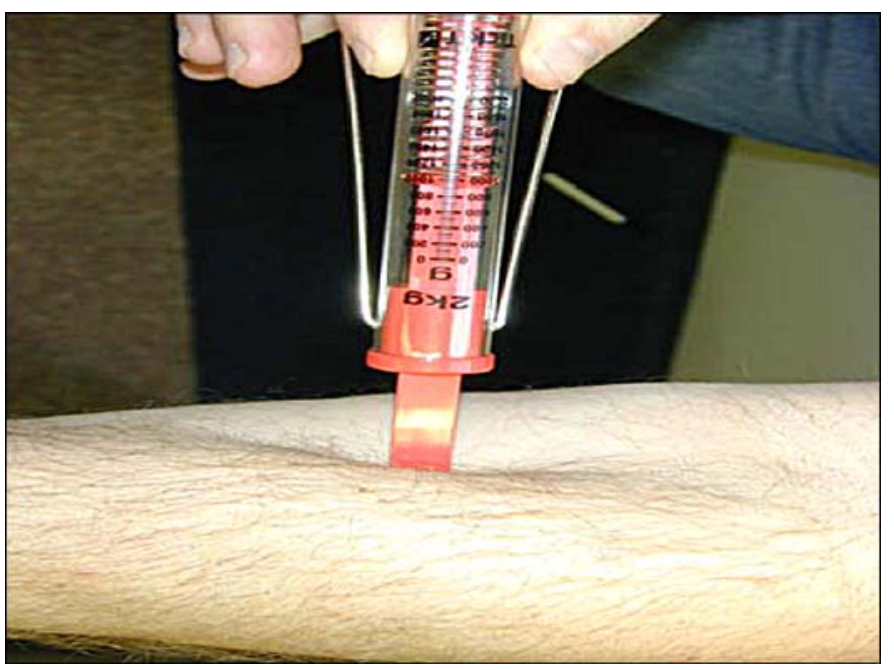

Figure 1.

Measuring tissue displacement versus applied force when disk is pushed into tissue of proximal forearm.

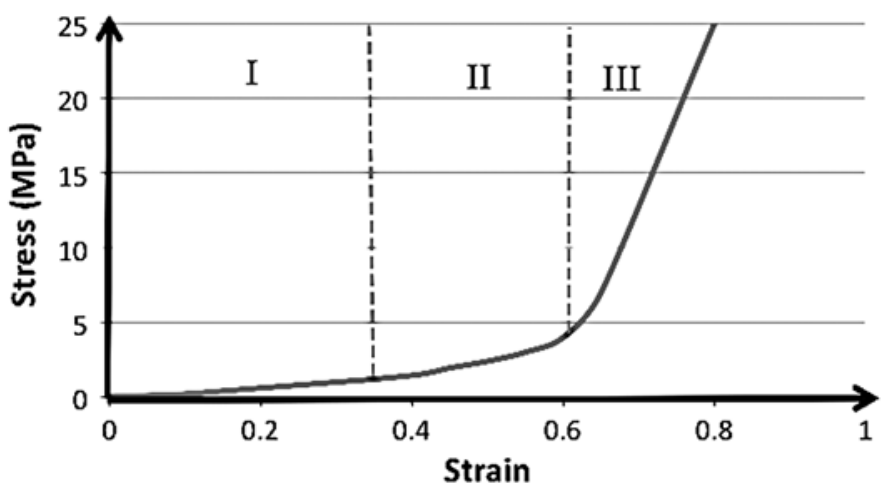

Figure 2.

Soft tissue stress-strain plot generated by data collected with an apparatus like that shown in Figure 1. Plot begins as straight line (area I), gradually slopes (area II), and becomes steep (area III).

discussion is sufficient for one to understand the theory of the CRS socket. Two 1971 articles fully discuss tissue compression [10-11]. When selected areas of the socket system are compressed to this degree, most of the lost motion between the limb bone and socket wall is eliminated. In the forearm and upper arm, the result will be greater user efficiency, increased lift capability, improved suspension, increased range of motion (ROM), improved stability, and improved positional capability. In a person with TF amputation, CSR will greatly improve gait efficiency (energy consumption, stride length symmetry, gait speed) because lost motion is eliminated. Balance when persons are standing will also improve.

\section{Tissue Compression in Tight Conventional Socket}

When the humerus is amputated mid-length and is then placed in a typical tight cylindrical socket, considerable potential prosthetic motion is lost when the limb tries to move the socket. This lost motion occurs because the end of the humerus must compress the muscle and fat between the bone and the socket wall before force can be transmitted. In the same way, the proximal socket must compress tissue before substantial torque can be applied to move the prosthesis. Two problems with a conventional socket must be considered. First, the forces are mainly applied only near the end of the humerus and to the bone under the brim, and second, considerable motion is lost before the limb can move the socket and prosthesis. Figure 3(a)-(b) illustrates what happens when a load is applied between a conventional socket and the underlying
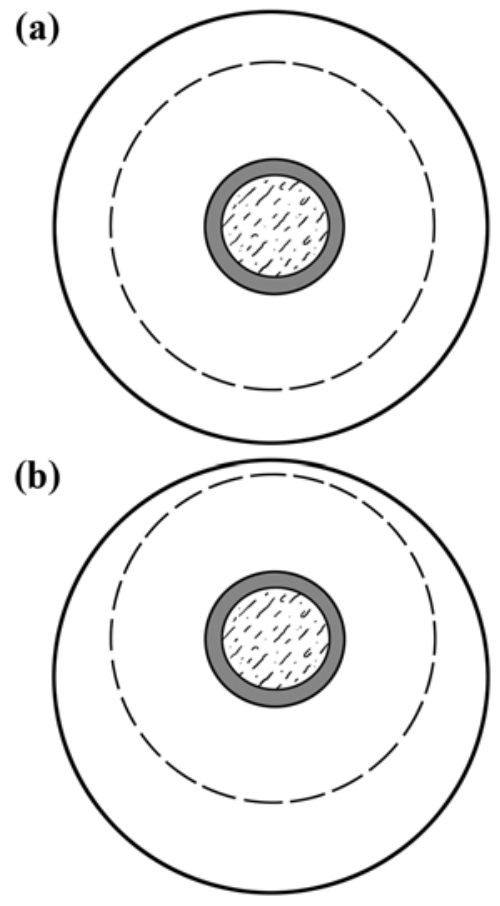

Figure 3.

Cross sections through distal end of conventional transhumeral socket showing why cylindrical socket loses motion. (a) Socket wall and humerus before application of force. Dashed line represents line of maximum compression. (b) Upward force has been applied to humerus causing it to move upward with respect to wall until tissue is compressed. Most of lost motion occurs before arm can cause socket to function usefully. 
tissue. Before the load is applied, the bone is centered in the socket. As a load is applied, the tissue must first be compressed until it reaches the level of compression to which no further motion is possible. In illustrations in Figure 3, Figures 4(a)-(c), and 5(a)-(d), the dashed line represents the level of compression to which no further motion is possible. Figure 3(b) shows how far the humerus must move before the prosthetic socket can support a substantial load.

\section{Use of Selective Compression to Stabilize Socket}

In a CRS socket, longitudinal depressions compress the tissue almost to the point at which no further motion is possible. This compression is possible because release areas are provided between the depressions for the displaced tissue to move into. Not every possible configuration of longitudinal depressions is optimal. The cross sections in Figure $\mathbf{4}$ show how two, three, and four depressions might compress the tissue, with the displaced tissue flowing outward. The two-depression configuration is stable in one plane only, while the other two configurations will resist motion in any plane. For anatomical, physiological, and other practical reasons, all sockets discussed in this article use four depressions.

Two reasons exist as to why the CRS socket increases the transfer of torque load from the user to the socket and the prosthesis. First and most importantly, the tissue is already compressed at both ends of the bone when a load is applied, so it cannot compress any further. Thus, load is transferred immediately with no lost motion. Second and less obvious, tissue is also precompressed along the entire shaft of the bone. Thus, even a small angular motion of the socket causes load transfer all along the bone, not just at the ends. In fact, a skilled prosthetist can adjust the amount of precompression to compress the tissue uniformly all along the bone when a full load is applied. Naturally, the actual transfer of torque is less near the center because the effective moment arm decreases as the center of the shaft is reached. Important to remember is that the tissue cannot be compressed without the tissue displaced. The tissue surrounding the longitudinal limb bones acts a little like the air inside a long balloon. When the balloon is pushed in at one point, increased air pressure moves it outward at another point. Thus, a key element of the new socket design is that it must accommodate the displaced tissue with longitudinal release areas. (a)

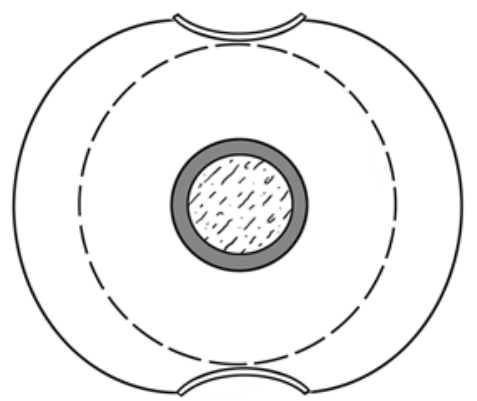

(b)

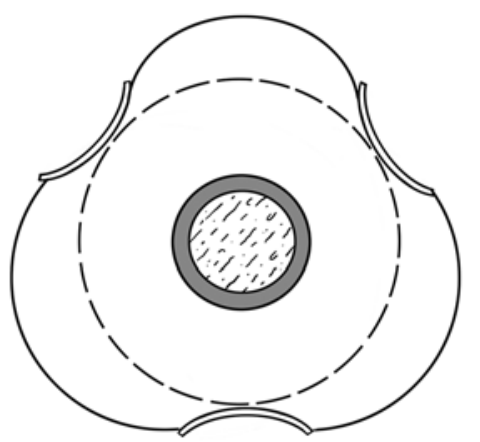

(c)

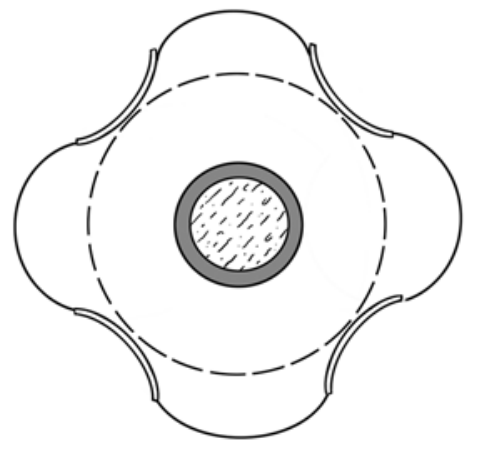

Figure 4.

Cross sections showing three designs for tissue compression by incorporating longitudinal depressions into socket: (a) two-depression design is only stable in one plane, but it will be used in Figure 5 to illustrate how compression/release stabilized socket works and (b) threedepression and (c) four-depression designs are both stable in all directions. Choice between them and their location depends primarily on limb size and functional envelope requirements. If limb circumference is so small that release areas do not allow sufficient tissue to flow into or through them to effectively take advantage of biomechanical principles of compression/release design, then three-depression approach may be necessary. Four-depression designs are most commonly used because of muscle belly location, functional requirements regarding range of motion, as well as any anticipated applied force (both magnitude and direction), whether internally or externally derived. In addition, local pressure tolerance of underlying tissue is also a factor.

\section{Selective Compression Reduces Skin Motion}

In a conventional socket, the smooth uniform surfaces fail to prevent motion of the skin, with respect to the 
(a)

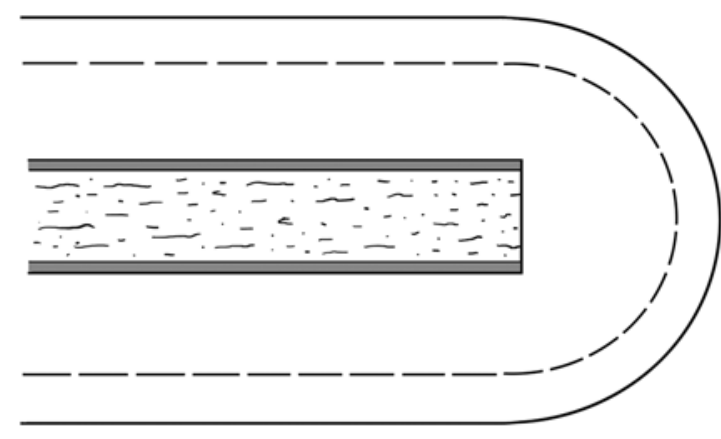

(c)

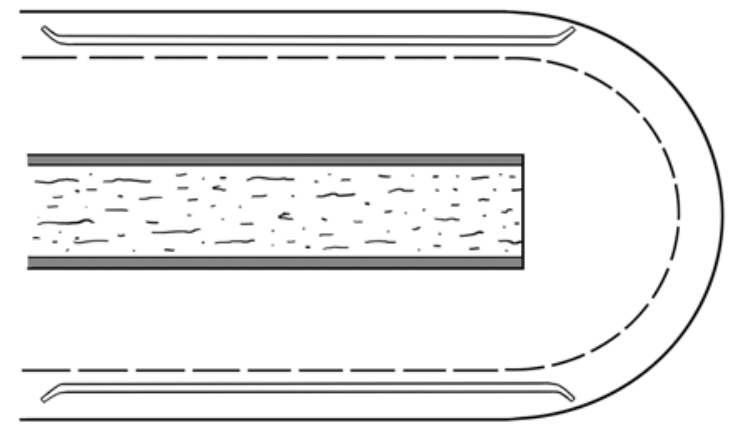

(b)

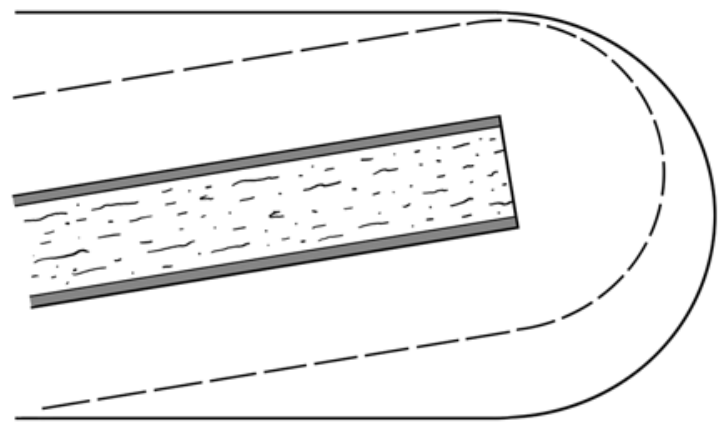

(d)

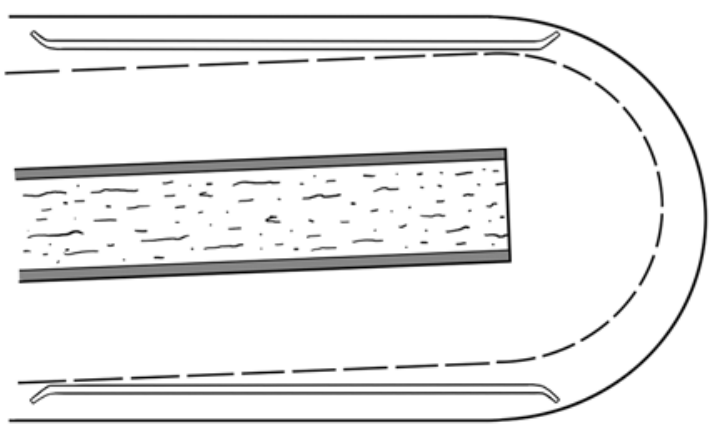

Figure 5.

Four longitudinal upper-arm cross sections show difference in how conventional and compression/release stabilized (CRS) sockets respond when arm tries to flex to transfer load to socket. In all four cross sections, dashed line represents level to which tissue must be compressed for load transfer. In conventional socket, (a) bone is centered before application of any load, but moves as shown in (b) second cross section when user moves humerus to flex arm. This action must first compress tissue at both ends as shown. Only after bone has moved through substantial angle will socket pick up load, and all load is carried at ends. In CRS socket, (c) cross section through two longitudinal depressions of Figure 4(a), tissue is already precompressed, so (d) fourth cross section load transfers almost immediately with little change in angle. Furthermore, load transfers along entire length of bone, not just at ends.

underlying tissue. Human limbs consist of bone covered by a layer of muscle, which is covered, in turn, by a layer of adipose tissue and skin. By several centimeters in any direction, the latter two layers are easily displaced longitudinally along the limb or rotationally around the limb before the connective tissue limits further motion. This easy motion of the outer layers explains why a prosthetic interface is so difficult to design. Friction alone between the skin and socket wall minimally stabilizes rotation or motion along the axis of the limb. With a CRS interface, the situation is quite different. The CRS longitudinal depressions and other indentations push into the underlying tissue. In addition to the primary function of the depressions that stabilize bone, a secondary function greatly reduces the motion of the outer layers with respect to the muscle and bone underneath. In a conventional socket, the interface wall in either a TH or TF socket is too far away from the underlying bone to use the shape of the bone to lock out unwanted longitudinal motion. However, when the tissue is selectively compressed, the compressed areas not only improve lift capability but also lock out skin motion. For instance, longitudinal depressions can extend close to the humeral or femoral head where they will restrict both longitudinal and rotational motions.

\section{Locating Longitudinal Depressions Circumferentially}

Consider the $\mathrm{TH}$ interface, which we will discuss shortly. Typically, the user needs maximal lift capability when the prosthetic elbow is at $90^{\circ}$ and a load is applied to the distal end. For accommodating this load, one may assume that two of the longitudinal depressions should be located on the anterior and posterior aspects of the interface. This assumption is wrong for two reasons. The first relates to stability. If the bone pushes directly against the inside of a depression, it tends to shift sideways and lose some of its stability (Figure 4(a)). If on the other hand, 
the depressions are rotated $45^{\circ}$, the bone will be trapped between two depressions. This configuration is much more stable. In addition, it will prevent the major nerves and blood vessels from compressing against either the humerus or femur.

Some important points to note are-

1. Stability requires three or more longitudinal depressions - four are usually best clinically.

2. Release areas adjacent to the longitudinal depressions must be adequate to allow tissue to displace between the depressions.

3. Before a load is applied, compression should be a little larger in the center of the bone. When compression is just right, loading the end of the socket will result in a uniform load from the center of the shaft to the loaded end.

\section{MOVING THEORY INTO PRACTICE}

The theory of the CSR socket just discussed can be readily applied to all amputation levels that use a socket or interface as well as many orthotic applications. In the following subsections are three examples of how the CRS theory could be applied. The evolution of previous $\mathrm{TH}$ and TR sockets is discussed in-depth by Andrew, Lake, and others [5-14].

\section{Transhumeral Compression/Release Stabilized Socket}

One can best understand the new CRS system for a patient with TH by following the fitting processes with a series of photographs. The socket developed as a sample is based on the configuration of Figure 4(c), which shows a cross section through the bone and the surrounding tissues, with four bars pushing inward to create the depressions. The width of these depressions will depend on the details of the patient's anatomy. They need to be large enough to spread the load but small enough to have sufficient room between adjacent edges for the displaced tissue to move outward. For a TH socket, the four depressions can be created as part of the cast-taking process. Figure 6 shows a simplified version of the planning or precasting stage of a typical fitting. Note that one precisely places the compression bars by carefully analyzing the limb and the functional demands of the patient. Figures 3 to $\mathbf{6}$ should be interpreted only as a simple representation of the CRS theory, intending to merely improve understanding of the concept rather than to use it as a blueprint for the fitting technique.

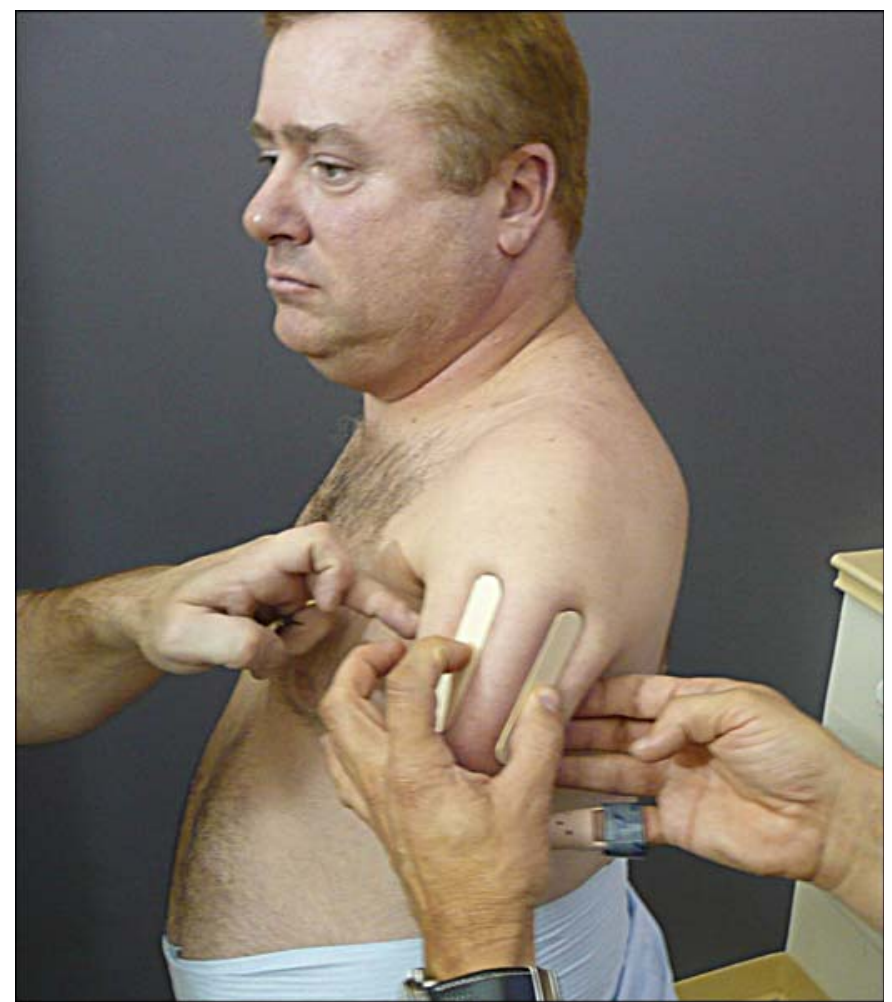

Figure 6.

Four modified tongue depressors represent actual compression bars for creating longitudinal depressions when cast is taken.

We selected tongue depressors to represent the specialized compression bars used to create the depressions and shaped the actual bars to include the appropriate contours for compressing the underlying humerus evenly. In this case, the tongue depressors have been shortened to suit the length of the residual limb. Two technicians are holding the tongue depressors in place while applying pressure. At this stage, the spacing between the compressed areas is important because it creates the release areas between the depressions, where tissue will displace outward. With this subject, the compressed areas are not uniformly spaced. A much wider opening can be found under the axilla and the posterior release areas than under the other two openings, which are spaced uniformly. This configuration maximizes stability in flexion and abduction, the most common combination of functional motions for the wearer of $\mathrm{TH}$, but reduces stability slightly during adduction and hyperextension because the forces of gravity aid the wearer during adduction and extension in most cases, and the need for hyperextension, though existent, is rare at this level. The wide opening also ensures a wide relief over the nerves and 
artery. After careful planning, the prosthetist takes the actual cast using a wrap of elastic plaster bandage with as little tension as possible. In Figure 7, the depressors are held in place, while the plaster sets to form the depressions. Figure 8 shows the depressions and release areas of the CRS socket. Figure 9 shows that additional compression is required. Figures 10 through 13 show the final stages of fitting another patient. Note particularly the size and location of the four depressions and the windows between them in the definitive socket. The antirotation wings are based on the original work of J. Thomas Andrew [5-6] but are smaller because of the added stability of the CRS design. To create these wings during casting, the prosthetist applies pressure in the anterior-posterior direction by placing the heel of one hand in the deltopectoral groove, with the other

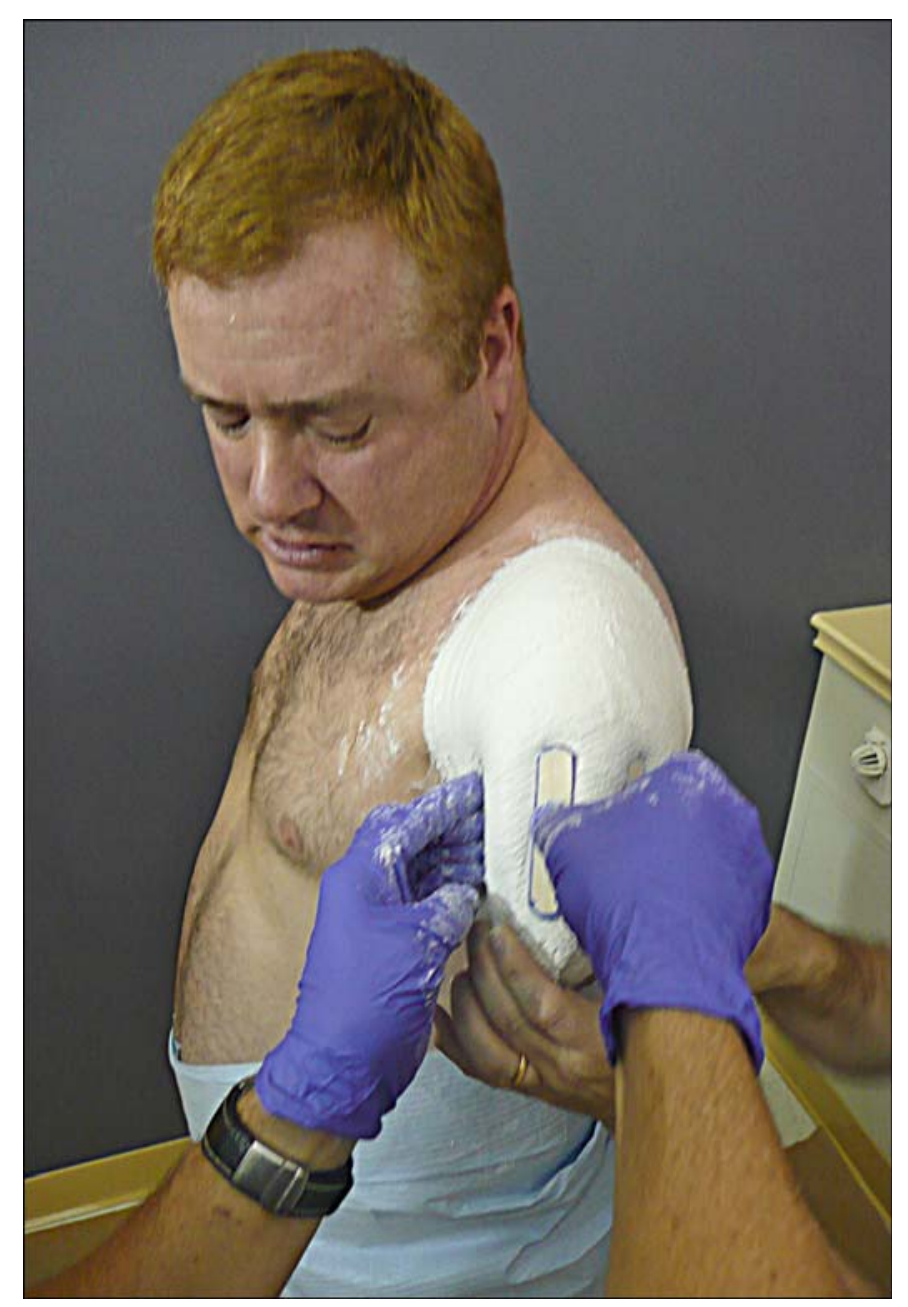

Figure 7.

Bars were outlined for visibility while being applied over very loose elastic wrap. hand over the posterior aspect of the spine of the scapula. In a fleshy patient, this pressure will precompress and additionally stabilize the tissues. While the area above the shoulder will be open in the final socket, one should connect the anterior and posterior wings with a band of plaster wrap to prevent spreading when creating the positive model. Typically, two persons will first use the bars to create the CRS portion of the cast, following which, splints are added and the wings are defined. See Figures 6-7 and 14-15 for fitting of participant.

We have added a second set of photographs to show other aspects of producing a TH socket. Figures $\mathbf{1 0}$ and $\mathbf{1 1}$ show stabilization wings, and Figures 12 and $\mathbf{1 3}$ show how to add myoelectrodes and a linear transducer to the definitive socket.

\section{Transfemoral Compression/Release Stabilized Socket}

What does stabilization mean for a person with TF amputation? The answer is that we need to address two stabilization problems. The first stabilization concern is to give the amputee control over the location of the distal femur. Typically, the surgeon should accomplish this stabilization by securing the distal aspects of the remaining muscles or tendons to the femur using techniques discussed by

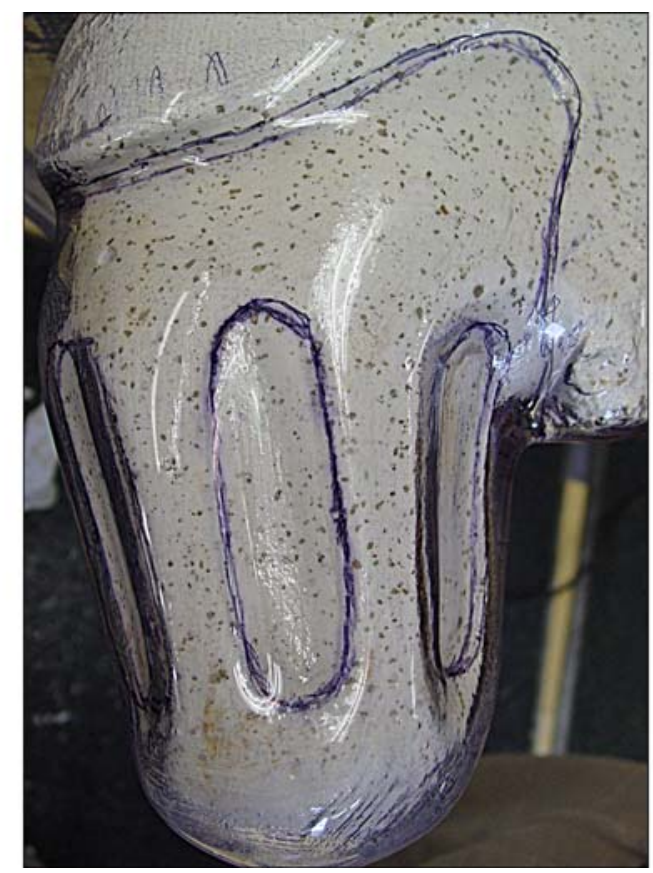

Figure 8.

Even though depressions have been deepened on plaster model before pulling first check socket, further modification will be needed. 


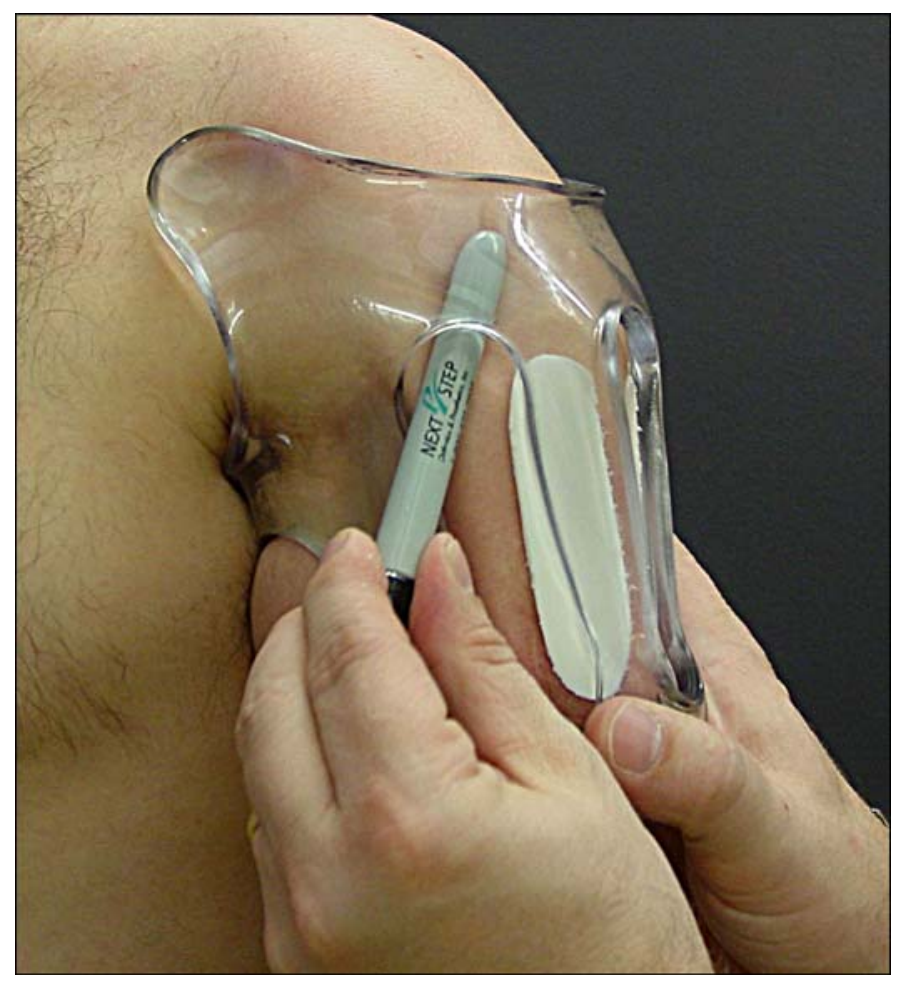

Figure 9.

Prosthetist added pads of stiff foam to increase compression and identify other problem areas to correct in next check socket.

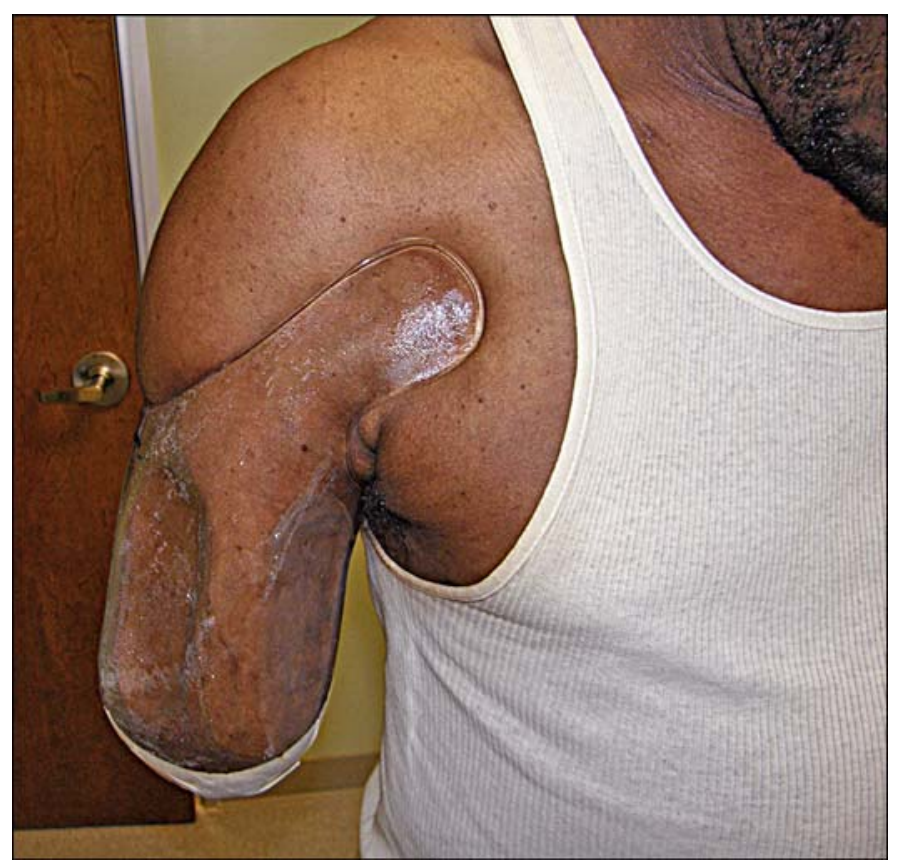

\section{Figure 10.}

Another user's check socket showing compression in deltopectoral groove.
Gottschalk [12]. When a surgeon performs a short TF amputation, the adductors, especially the adductor magnus, are the most difficult to secure but are necessary for stabilization. Another answer is that the socket and in turn the prosthesis must be stabilized with respect to the femur. For this stabilization, the new CRS socket excels. Typically, a TF

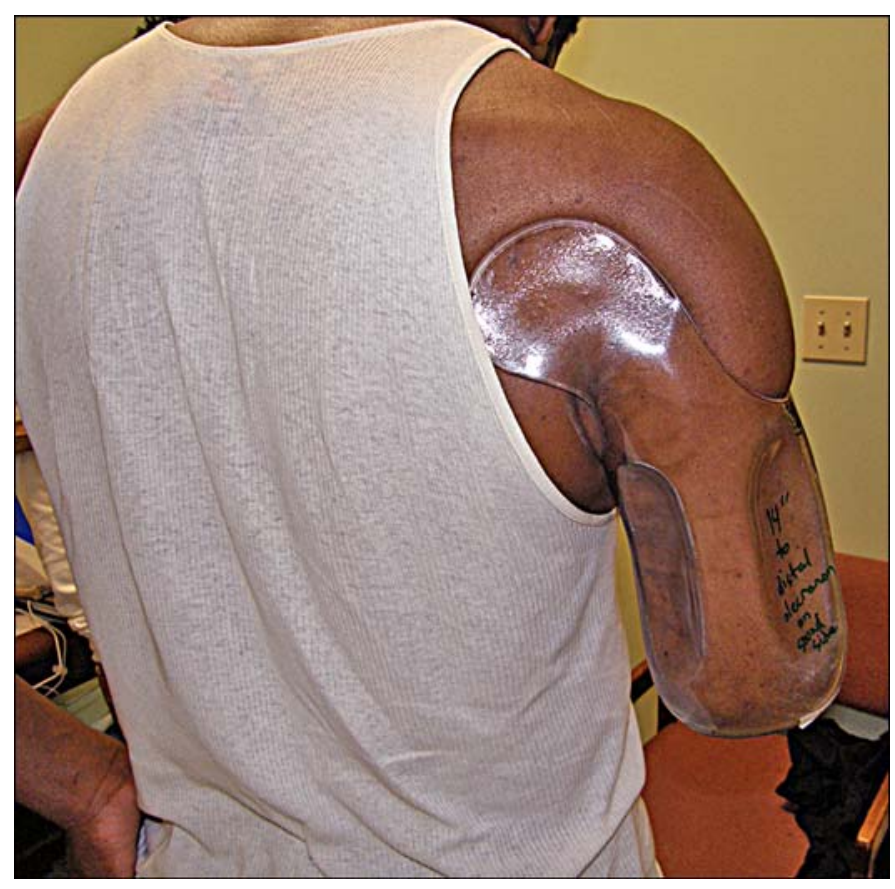

Figure 11.

Socket of Figure 10 showing posterior stabilization wing displacing tissue.

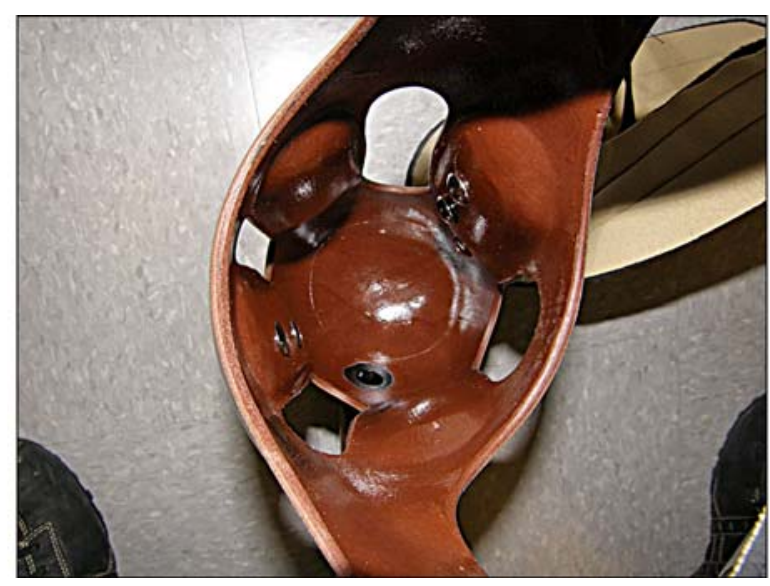

Figure 12.

Definitive version of interface shown in Figures 10 and 11. Note substantial depressions with pairs of myoelectrodes mounted on two of them. 
socket will follow well-established principles on the proximal end, including those introduced with recent subischial designs [13]. To turn a typical TF socket into a CRS socket, compressive stabilizing forces must be directed toward the femoral shaft through its entire length. This stabilization is accomplished by four longitudinal depressions with release areas added between them. With these depressions, the femur is captured throughout its range, ischial containment becomes unnecessary, and distal end pressure concerns are mitigated because of the added capability of the interface to control motion of the femur in any direction within the socket, including longitudinal motion. In addition, sitting comfort is increased because the entire structure no longer needs to extend as far proximally, particularly in the anterior and posterior regions. Some newer well-known TF sockets promote the benefits of greater control due to the taller proportions of the socket; however, an emphasis on proximal control is necessary only because of the lack of adequate stabilizing forces provided by these designs. The CRS sockets described in the following paragraphs not only stabilize the

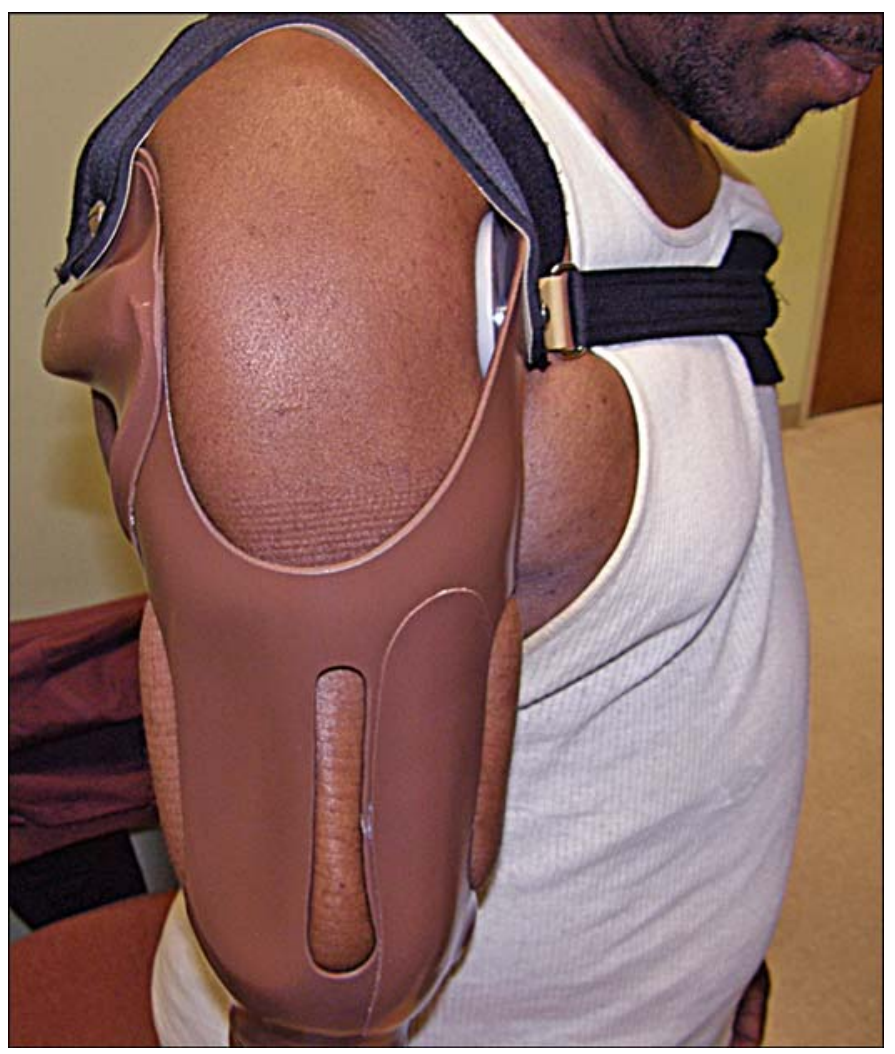

Figure 13.

Subject is wearing definitive socket. Note soft tissue flowing out of release windows.

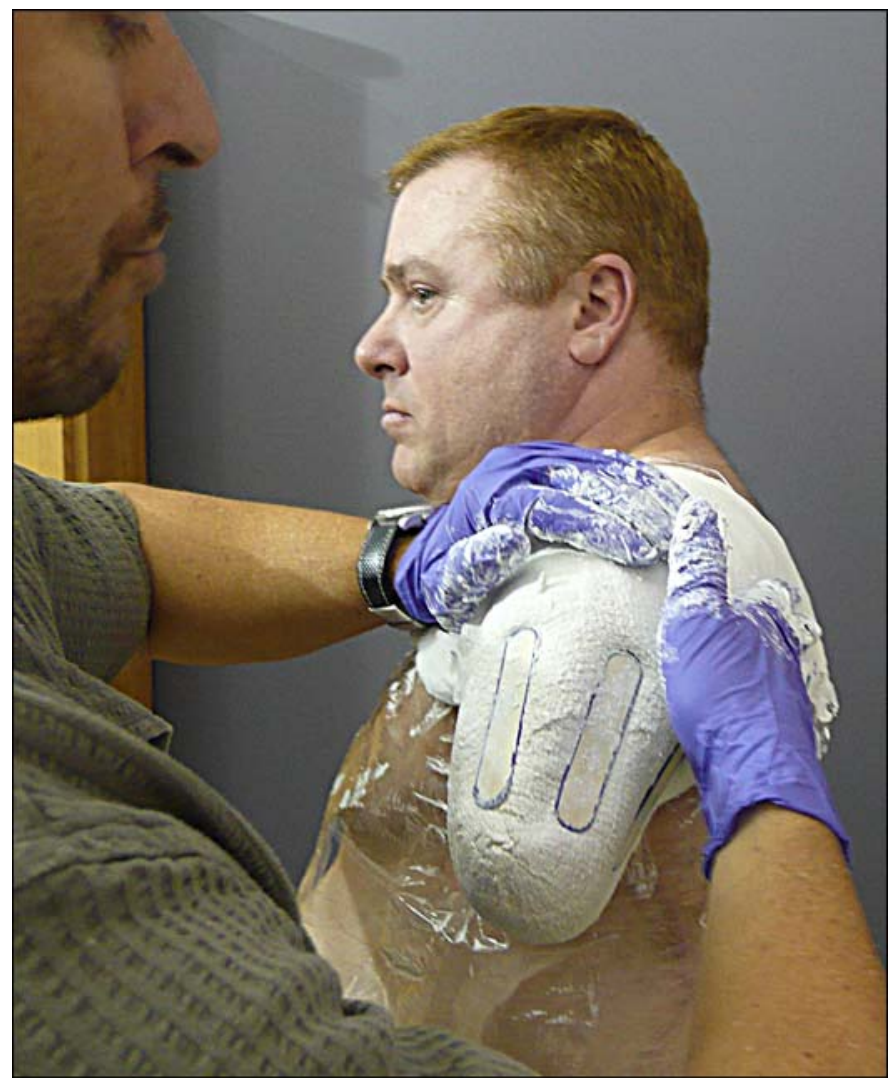

Figure 14.

Prosthetist added additional plaster splints across shoulder to permit compression in anterior-posterior direction to create antirotation wings.

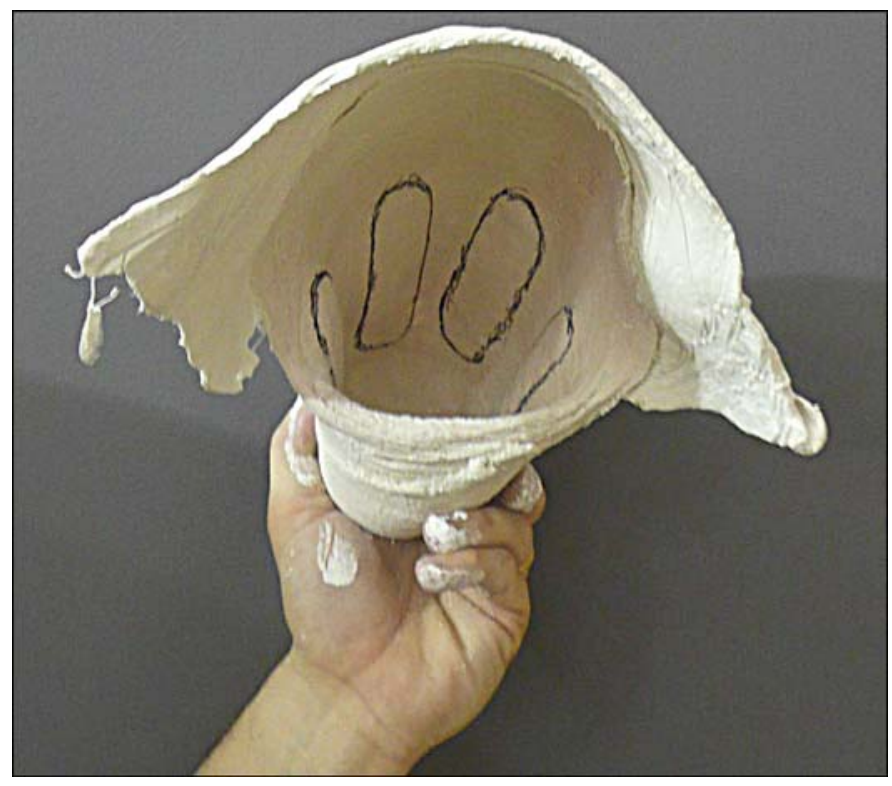

Figure 15.

Depressions have been outlined inside cast. 
femur but also provide a cooler and more comfortable interface by using a stiff carbon-laminate frame with large open areas. Important to note is that the temperature of the skin varies from frame to window in the CRS sockets even when a silicone liner is used.

A positive model for laminating a TF frame interface is created in three ways. The first is essentially the method just discussed for the TH interface; however, because the thigh is too large for the hands alone to compress the four longitudinal pads adequately, a specialized jig for aligning and compressing the pads has been created. We have designed such a jig that is now being tested on selected patients as part of a study that will appear later, and once the training program has been completed, it will be provided to those certified in the design.

Without a jig (the second approach), one can create the depressions by pushing inward against four large pads with an elastic wrap; however, this technique is not recommended for a variety of reasons regarding safety, efficacy, and proper alignment. We discuss it here merely as an example of how we first applied the technique. The pads must be thick and rigid enough so that they will allow the elastic wrap to bridge the release areas. The reader can see how large these pads need to be by studying the longitudinal depressions in Figures $\mathbf{1 6}$ to 20.

A third approach is to use a laser-scanning tool to create an exact replica of the exterior of the soft tissue either loaded or unloaded. We have developed a casting jig system to increase precision during the casting process and will be working with computer-aided design systems and their manufacturers to develop the appropriate tools for digitizing and fabricating the high-fidelity interface. When this model is placed on a computer screen with suitable software, the socket can be modified that results in a good first check socket. Rules can be built into the software to modify sockets consistently from patient to patient. For instance, the overall shape can be reduced by a percentage to create a tighter interface. More importantly, the software can be set up to keep the area through any cross section at its initial value or at a value that is reduced by an exact percentage. Such a rule automatically generates the required release areas when the depressions are generated. Some work has been done on creating this software, but again, it is not yet ready. The prosthetist fabricating a TF CRS socket for the first time will benefit from using the casting jig to create the depressions in a traditional plaster wrap. The experience gained will be invaluable when suitable software becomes available.
Following the creation of a TF frame interface from a modified plaster model to a finished frame to be worn by an amputee is useful. Figures $\mathbf{1 6}$ and $\mathbf{1 7}$ show an early version of a rectified plaster model and the clear plastic check frame thermoformed over it. Figure 18 shows this check frame being evaluated on the patient for whom it was made. A few minor modifications will be needed when it is used to create the final frame interface. An early

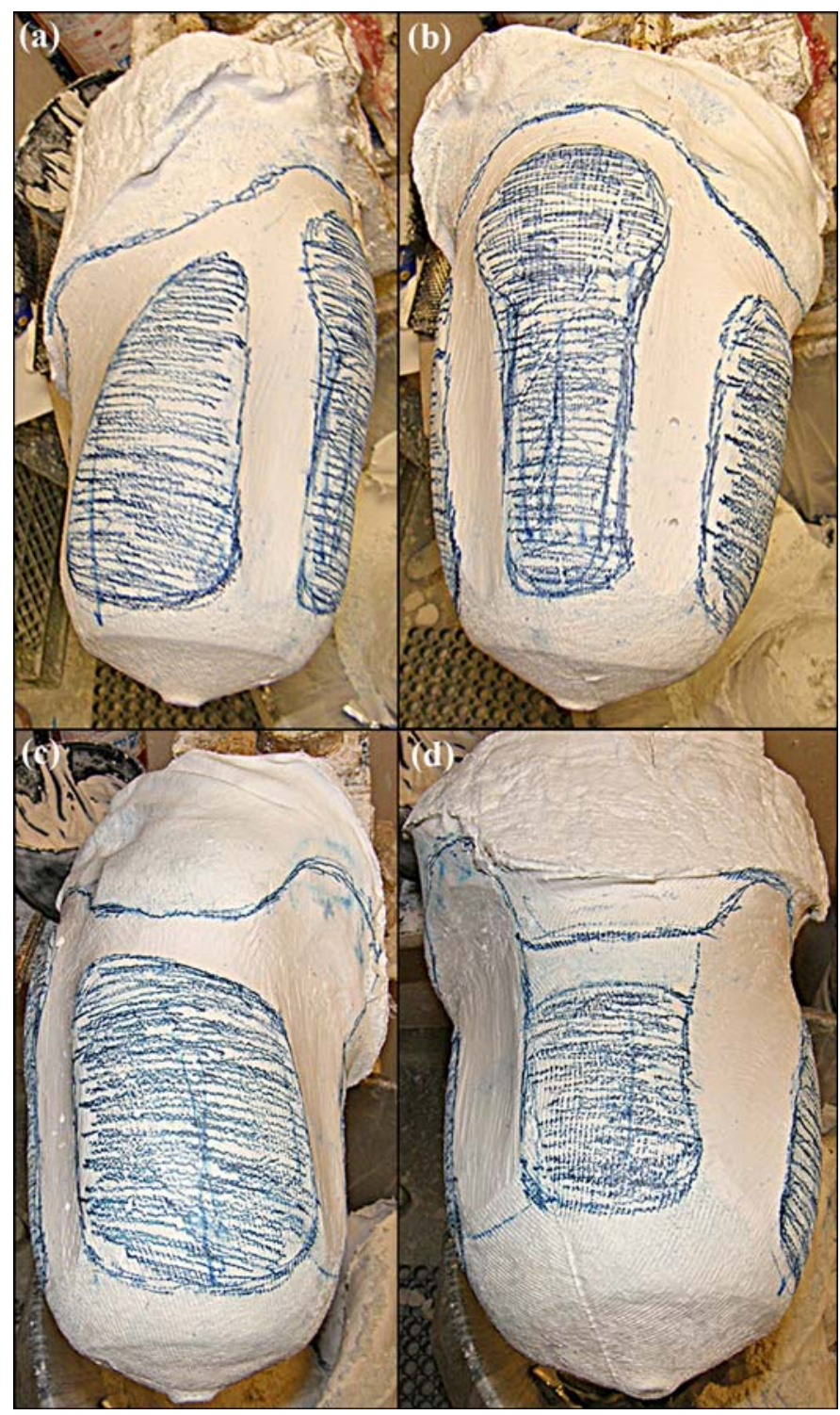

Figure 16.

Four views of rectified transfemoral model showing high release areas (colored with an indelible marker) with smooth longitudinal depressions between them: (a) medial, (b) anterior, (c) lateral, and (d) posterior aspects. 


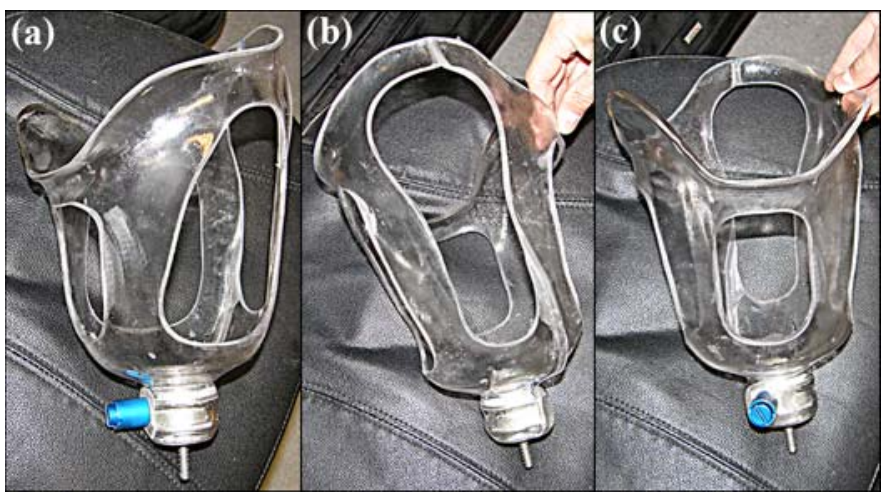

Figure 17.

Check socket with pin-lock mechanism made over Figure 16 model: (a) posterior and medial reliefs and (b) lateral and (c) posterior releases.

version of a finished frame with the distal components are shown in Figure 19, and a second is shown in Figure 20, along with a frame being tested on or by the patient. The photographs shown are from an early fitting of over 30 clinical fittings done by Alley and his team. The design has changed considerably since then, but the images shown are intended to represent the CRS principles. Of special note is that while pin suspension has been shown to excessively distend or pull distal tissues in traditional sockets, in the CRS socket, this is mitigated, and no complaints of this common problem have been noted. This result is believed to be from the added friction of the com- pression bars along the length of the liner as well as the added suspension that the escaping liner provides through the release windows. The result of further studies of this phenomenon will be published subsequently.

\section{Transradial Compression/Release Stabilized Socket Systems}

The full literature on the development of TR sockets can be found in a study by Lake [7]. In many cases, recent improvements were possible because of the new materials or techniques available to make them practical. These improvements began with the new lamination techniques developed during World War II. Gradually improved resins became available at a reasonable cost, and the stockinet for reinforcement became both stiffer and stronger. At the same time, thermoform plastics became available for the rapid production of check sockets. Later, elastomeric liners were introduced to increase suspension and comfort for the person with a lower-limb amputation, and these were soon adopted for those with the upper-limb amputation. In addition, extremely pliable thermoformed liners replaced rigid inner sockets in many prostheses. As a result, today, many practitioners are using sockets with openings that are bridged only by thin flexible membranes. TR sockets have benefited from all of these improved technologies.

Prosthetists have been working for many years to optimize self-suspending TR sockets. The Muenster [1] and Northwestern [2] designs were developed with the advent

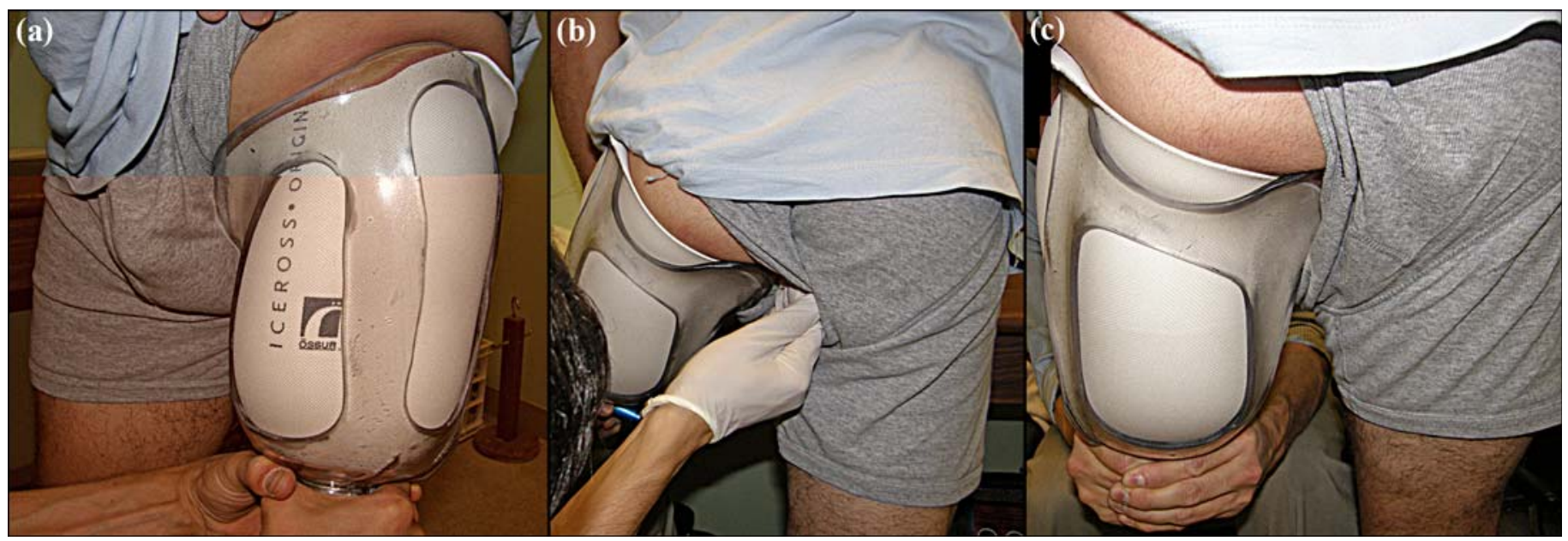

Figure 18.

Socket of Figure 17 attached to distal mechanisms and tried on patient: (a) anterior-lateral view, (b) checking medial flare, and (c) posterior view. In images (a) and (c), prosthetist is applying force to check stability. Socket shown is early generation of transfemoral design. Subsequent design iterations have significantly lowered proximal trim line. 


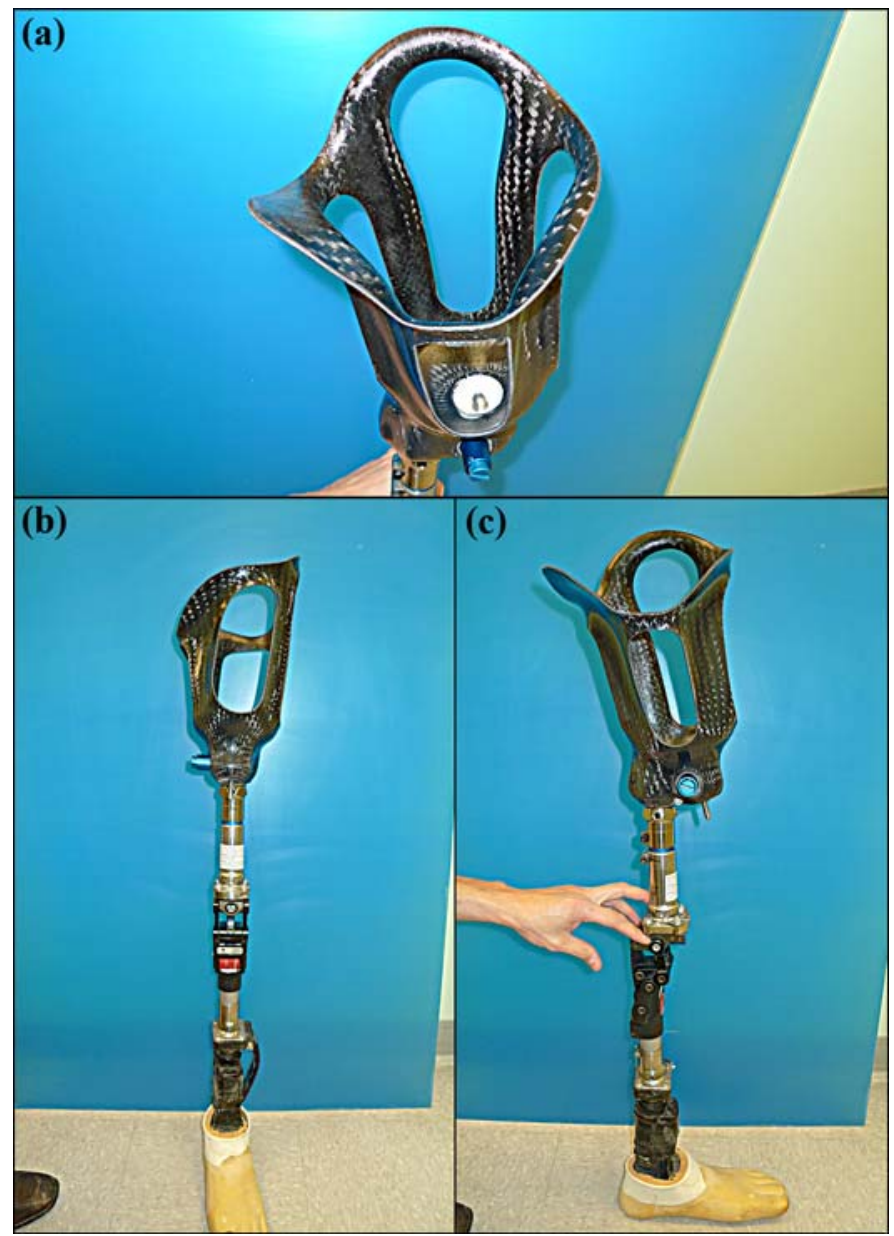

Figure 19.

Three views of same left compression/release stabilized frameinterface transfemoral socket: (a) medial, (b) front, and (c) lateral. Note how frame edges are contoured for comfort. User will don thin soft liner between skin and frame.

of myoelectric control, which eliminated the need for a control harness. Both of these designs have shortcomings. To address these shortcomings, a number of presentations were made at meetings and symposia in the early 1990s without being reported in the peer-reviewed literature. For instance, in 1995, Alley began experimenting with the anatomically contoured and controlled interface (ACCI) on a patient with a very short TR amputation, and in 2002, he published the results of this work [8]. The best description of the ACCI, which clearly shows how depressions are used on either side of the radius, is given in another work [9]. This 1995 design was in response to an earlier design referred to by some as the Anatomically Contoured Socket, which lacked these radial depressions and there-

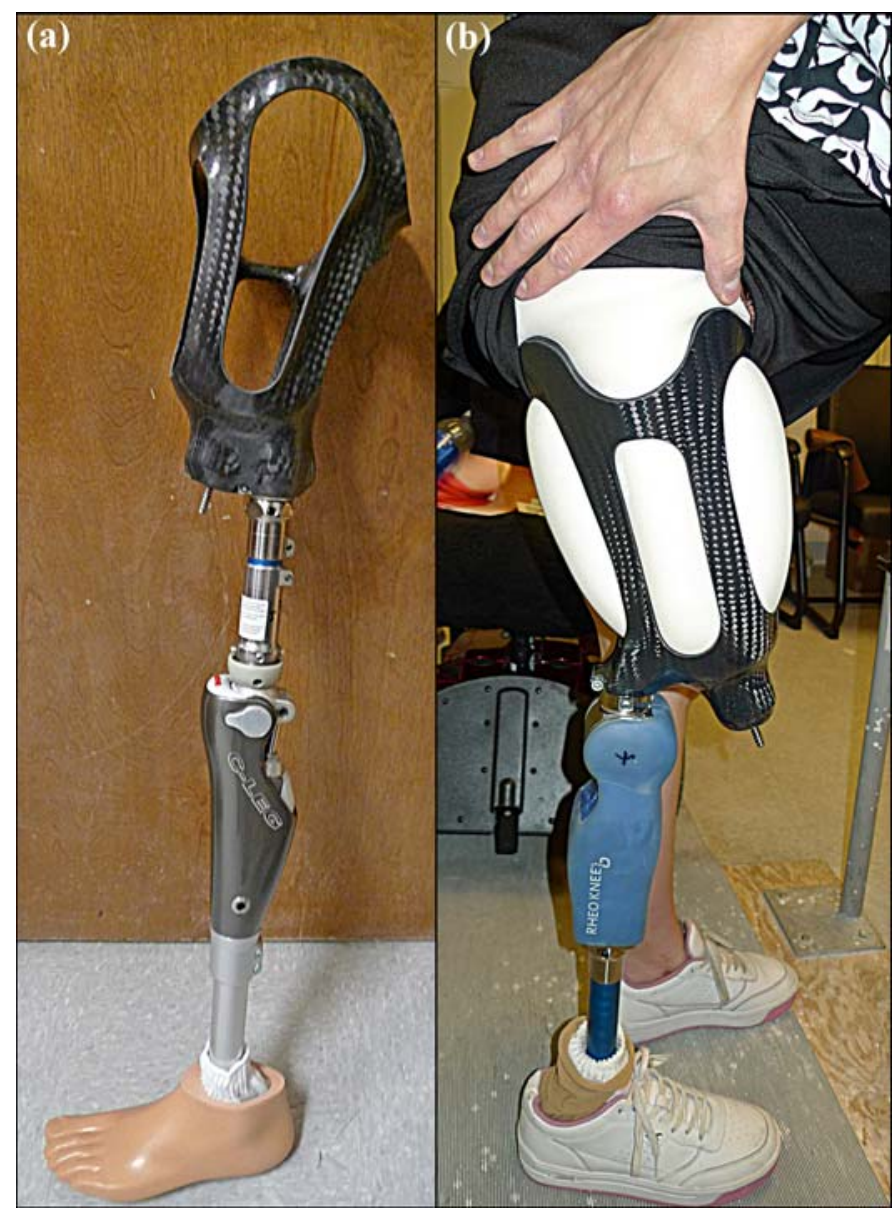

\section{Figure 20.}

More compression/release stabilized (CRS) frame-interface transfemoral prostheses: (a) short frame for thin patient with offset locking pin and (b) accommodating long femoral remnant with severe flexion contracture. CRS socket allowed patient to walk again after being confined to wheelchair $>10$ years.

fore allowed excessive bony motion and provided limited functional surface area during the initial stages of flexion. In 2003, Miguelez et al. described the Transradial Anatomically Contoured (better known as TRAC) socket [14], modeled after the ACCI. These designs concentrated on addressing the fit and relief of particular areas on the remaining limb through contouring to accommodate the underlying anatomical structures. In particular, they created a compartment distal to the cubital fold to receive displaced tissue during elbow flexion. This compartment addresses a problem with both the Muenster and Northwestern designs in which tissue often bulges distal to the cubital fold when the amputee flexes beyond $90^{\circ}$. In addition, both designs address the problem of discomfort just 
proximal to the epicondyles. The common element is moving the proximal trim line entirely into the cubital fold, where it is contoured to accommodate the biceps tendon and bicipital aponeurosis. In the ACCI, depressions on either side of the radius apply precompression, and a pocket is provided distal to the anterior trim line to accommodate the tissue displaced by these depressions as well as the tissue displaced during full flexion. These changes to the traditional designs increase the flexion range and add comfort under load during lift. The depressions also increase lift capability. While these depressions help with lift and stabilization, according to the CRS principle, they are only fully effective if adequate relief is provided for the displaced tissue. Thus, Alley's TR CRS socket has added increased soft tissue release and multiple depressions for further stabilization. The CRS design concentrates on adding stabilization of the bones along their entire length not just in a few areas, but the TR socket also retains the contouring of the other designs, which is important for permitting a full ROM with adequate comfort.

The explicit addition of release areas allows the prosthetist to change the traditional double-wall socket into an open-frame interface. Typically, the load-bearing structures become stiff carbon fiber struts and brim structures, with the remaining tissue covered by only a thin flexible membrane that replaces the traditional hard inner socket. In some areas, even the membrane can be eliminated for enhanced cooling and suspension. The four longitudinal depressions in the latest design lock the radius and ulna along their entire length while the release areas between them receive the displaced soft tissue. Merely adding room for muscle hypertrophy as is commonly lauded as a design feature in many sockets is insufficient to stabilize the bones. The four depressions also prevent the rotational instability present in prior designs, allowing for a much lower proximal trim.

Carbon-fiber frame interfaces are ideal for heavyduty users. Simple frame interfaces are also appropriate for extra-lightweight prostheses for children. The tubular Wilmer frames introduced by Plettenberg [15-16] in the Netherlands share the windowed structure inherent in the CRS design but without a focus on enhanced compression through alternating release of soft tissue.

\section{Sample Fittings of Transradial Compression/Release Stabilized Interface}

The principal features of a CRS interface for the person with TR amputation are shown in the following discussion. Figure 21(a) shows a solid model of an Alley
TR socket design with four longitudinal depressions, two of which are visible. The two on the upper surface, with release between them, stabilize the anterior or upper aspect of the radioulnar complex, while the lower pair stabilize the posterior or lower aspect. The ulnar compression bars are new with this design, though important to note is that the level of compression of the radial bars is much greater than the level of compression introduced in the earlier ACCI design due to the release of soft tissue in the CRS socket. The ulnar compression bars provide a large stable surface area just distal to the olecranon to counteract the force on the distal end of the radial remnant when a load is lifted. Note that the olecranon itself is free. This innovation was introduced in 1986 by Sauter et al. [17]. Figure 21(b) shows how even a short residual limb can be provided with depressions on either side of the radius. The development of a patient's

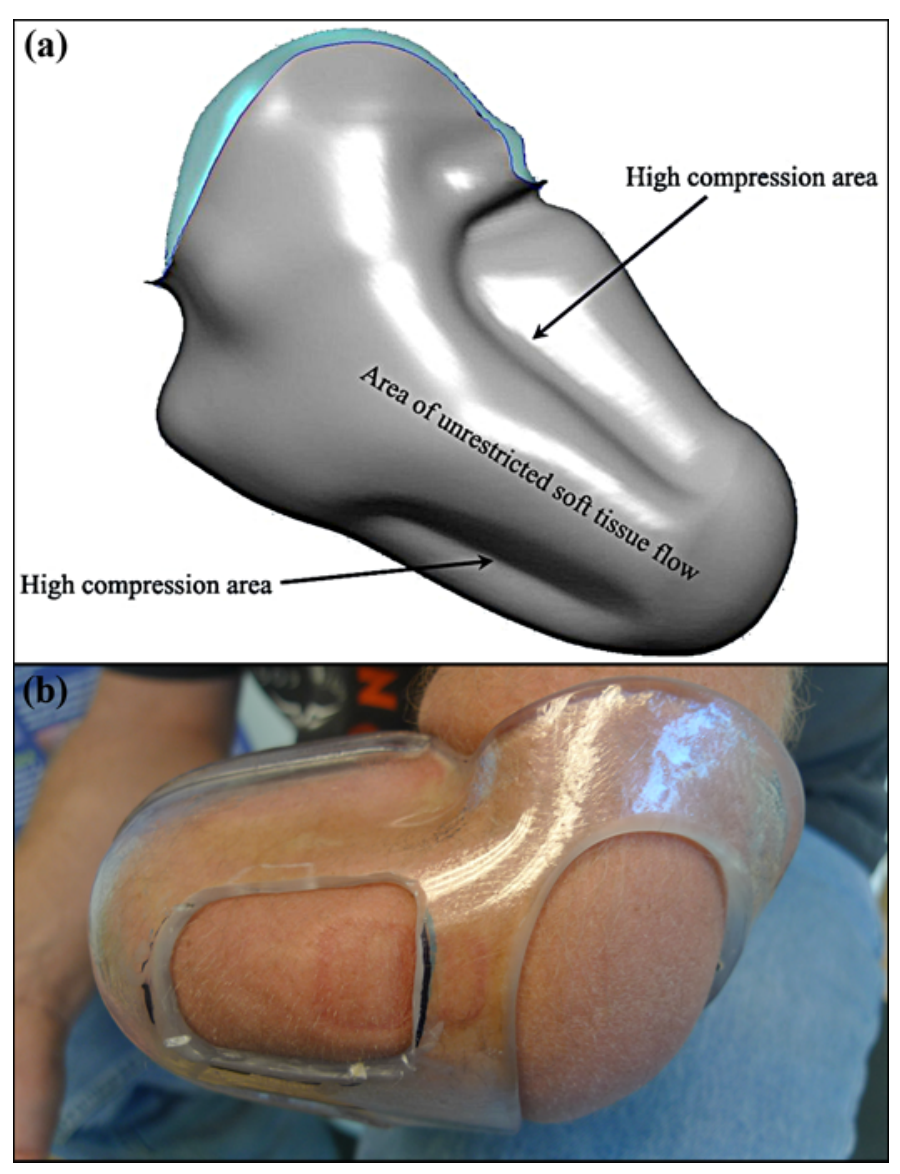

Figure 21.

(a) Computer model of short compression-stabilized radial interface. (b) Note similarity of actual short transradial check socket to model in (a). 
socket starts with a traditional cast that is modified by adding the CRS features. Figure 22(a)-(d) illustrates a typical TR fitting process. The release areas are provided during the development of the socket, but in this particular case, the definitive socket is closed to provide mounting areas for myoelectrodes. The fully open frame shown in Figures 23 and $\mathbf{2 4}$ does not need these mounting areas. Were this patient to require myoelectric control, electrodes could be added to a thin liner or a single strap (with elastomeric qualities) sufficient to mount the electrode. Placing an individual metal electrode into a roll-on liner is easy; however, transferring the myoelectric signal to a preamplifier is difficult. Daly introduced the first successful roll-on liners with myoelectric pickups in 2002 [18], but this problem currently needs additional research and development.

\section{DISCUSSION}

This article introduces a new conceptual framework to help us understand the design of prosthetic socket interfaces for three of the major amputation classes. With the framework so new, no scientific proof currently exists that the technique discussed will be a better design for patients than conventional designs, although Alley is

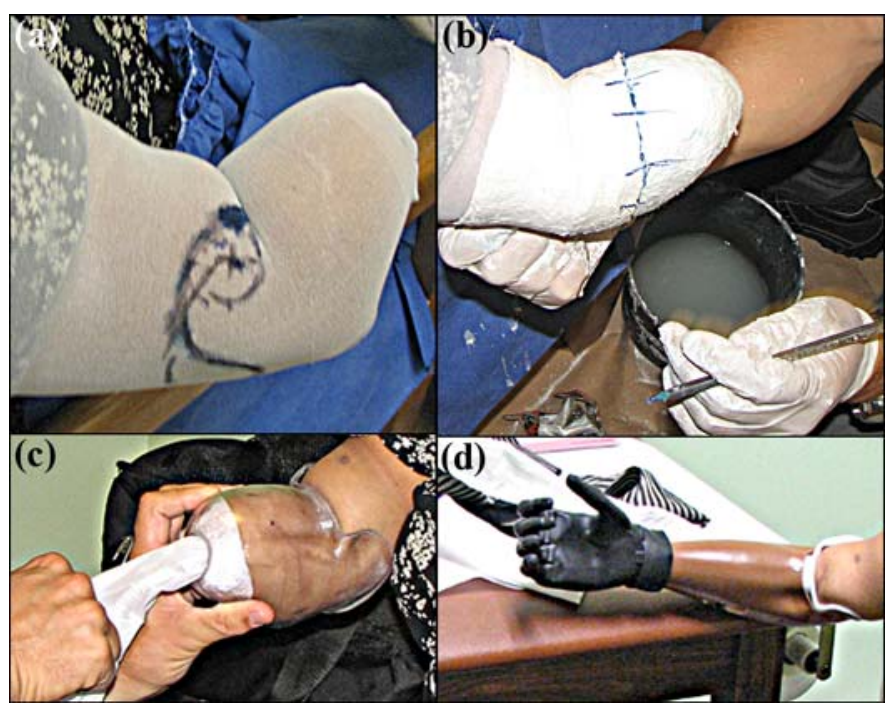

Figure 22.

Four stages in fitting of compression/release stabilized prosthesis to short transradial residuum: (a) Stockinet marking, (b) cast application, (c) depressed areas in check socket, and (d) definitive with short depression. currently developing the protocol to be used in multiple clinical studies. The new technique meets one important criterion for application of the word scientific, however. It is supported by actual measurements that validate the concept underlying the technique. Figure 1 shows how tissue displaces during local compression and explains why negligible motion is lost as shown in the transition from Figure 5(c) to 5(d). To date, no known contraindications exist except hypersensitivity at the precise locations of the compression bars. And yet even in this case, the compression bar length, width, or magnitude can typically be adjusted to account for regional sensitivities.

The best science eliminates as many variables as possible. Funding a study where every amputee gets two prostheses is difficult. One way around this dilemma would be for the new master's-level prosthetic programs to test both conventional and CRS sockets made by students for the same subject as part of their training. Doing such a study should not significantly increase the cost of such a program, and it would produce significant data. Naturally, the students would need to be properly trained and certified in the design and only well-fabricated samples should be used in user comparisons. The authors are presently pursuing research and considering both certification and licensure for clinical training.

Local pressure is not a new problem in prosthetics. However, when the socket purposely applies local pressure, ensuring that this pressure does not reduce the perfusion of blood in the underlying tissues is important. We have been studying the relationship of contact pressure and tissue perfusion under static surfaces and in the released tissue in the windows. Although the pressure data in the wheelchair literature are related, our socket interface with strategic regions of compression and release is different mechanically and physiologically. The important point is that if contact pressure exceeds the capillary perfusion pressure, blood flow will stop and the tissue will become ischemic. Although the soles of the feet and the gluteal regions are subject to high pressure, the pressure is intermittent and not sustained for 8 to 12 hours as might occur in a prosthetic socket. The goal when studying perfusion is to find a static preload pressure that allows adequate blood flow and is not excessive.

What is adequate blood flow? While uncompromised blood flow with zero pressure on the skin would be optimal, this does not sufficiently preload the soft tissue to provide the biomechanical advantages inherent in the CRS socket. Additionally, within any prosthetic interface 


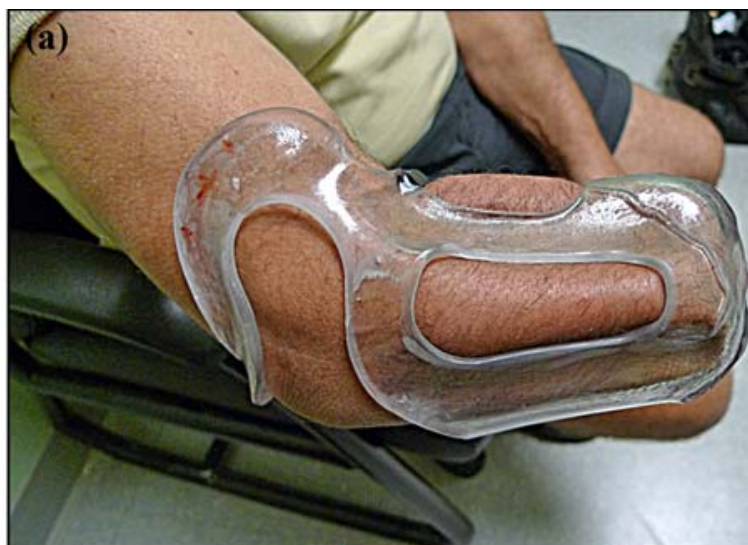

(c)
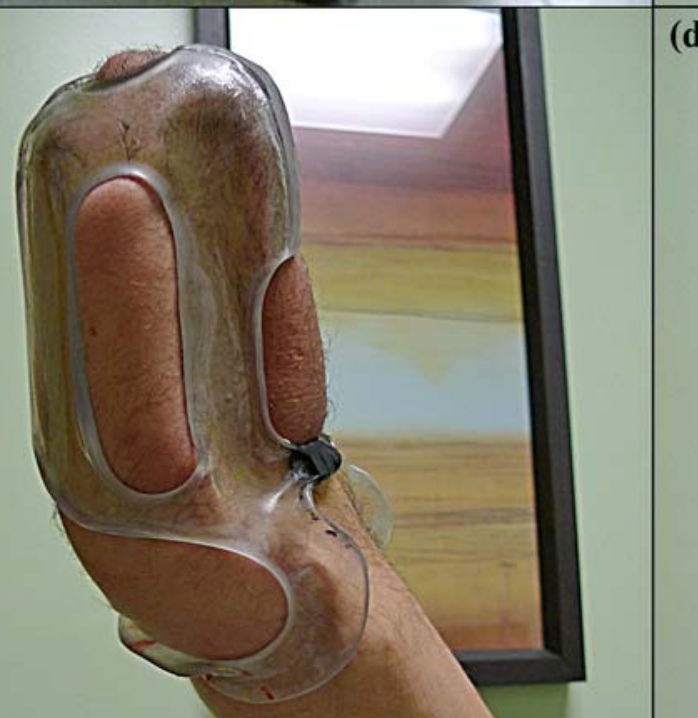

(b)

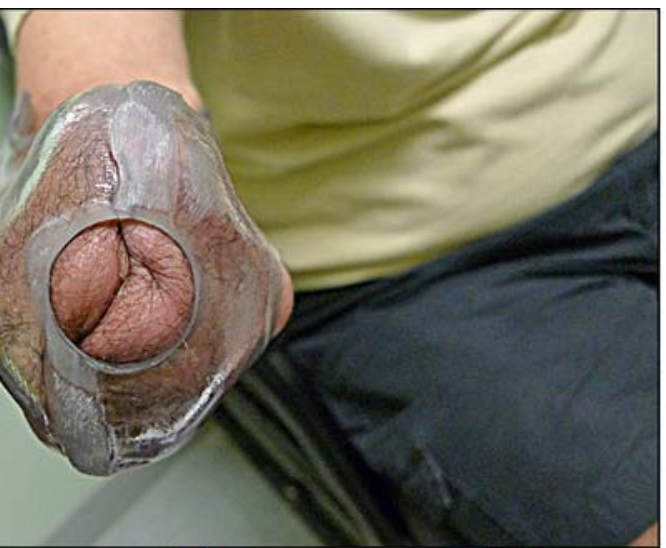

(d)
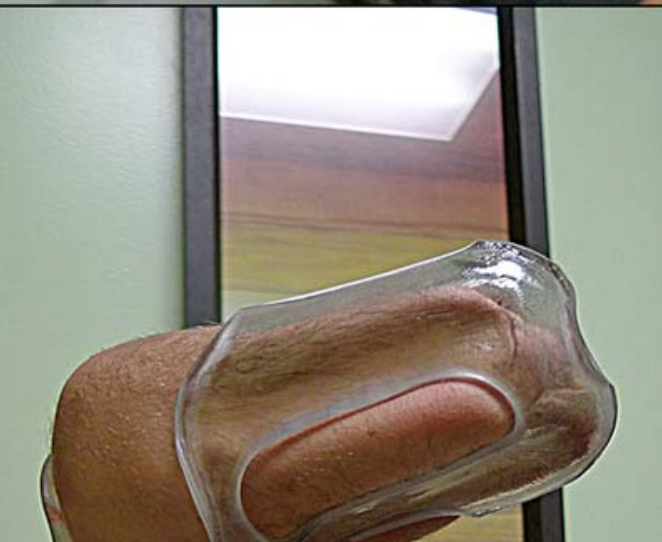

र

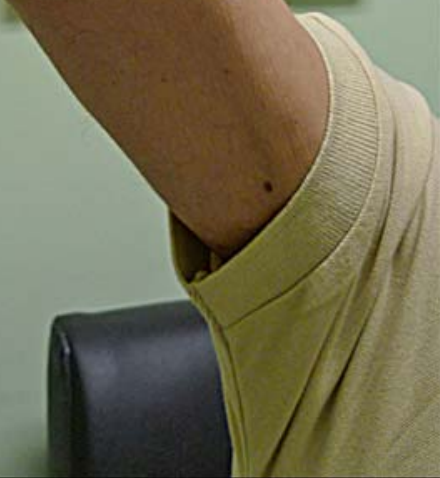

\section{Figure 23.}

This relatively long check socket clearly shows four depressions that form longitudinal stabilizers: (a) lateral, (b) distal end, and (c)-(d) medial views. Openings appear wherever compression is not required.

where the skin meets an interface wall, zero pressure at the skin is simply not possible. At the other extreme, however, fully compromised or zero blood flow is obviously not an option. The answer lies somewhere between these two extremes.
Finding an acceptable perfusion level without complex instrumentation is not easy for the experienced prosthetist. Use of the postischemic hyperemia response (redness of the skin after removal of the prosthesis) is one approach to finding the optimal pressure. The magnitude 


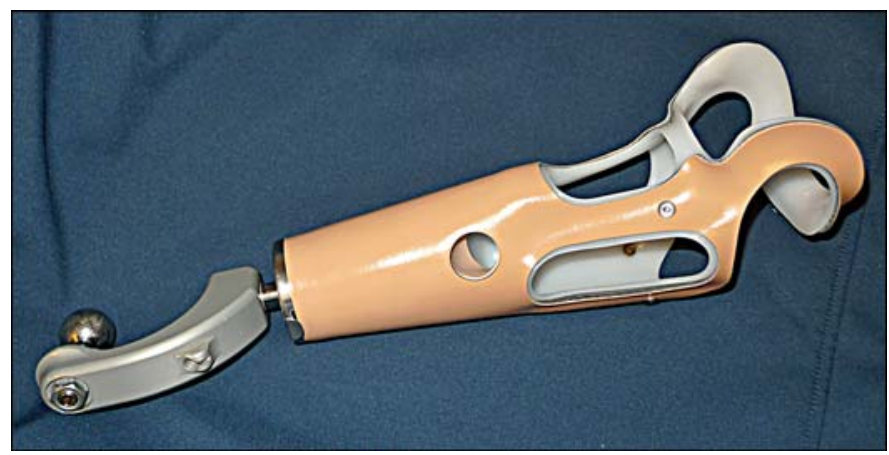

Figure 24.

Definitive prosthesis made using check socket of Figure 23 with special-purpose terminal device for cycling and boating.

and duration of this response are good in measuring how much oxygen and metabolic deficit the tissue has experienced. With conventional prosthetics, this redness takes around an hour to subside. In the more advanced socket designs with greater pressures, reduction of redness can take 3 to 4 or more hours, after wearing the prosthesis for 3 to 4 hours. An important development of our research has begun to show that the body actually adapts over time to increased compression by pushing more blood into the area affected. In addition, an active patient may mechanically "pump" fluid in the compressed tissue with movement and contraction, and the thresholds for redness will be different. The patient with lower-limb amputation will have different considerations, such as mechanical or hydraulic, and tissue capillary pressures will be greater (perfusion pressure is equal to blood pressure plus the pressure of the column of blood between the heart position and the leg site). Thus, to ensure safety, both the prosthetist and the patient need to monitor how long redness persists after the prosthesis is removed. If it persists more than 3 to 4 hours, the prosthetist needs to reduce the level of compression either by moving the compressing member further from the bone or by making the member wider to spread out the force.

Additional research is ongoing to quantify acceptable pressure levels. While a study will use advanced instrumentation, the goal is to give the prosthetist certified in this design simple tools for protecting the patient from harm while delivering the increased stability inherent in the CRS interface system.

\section{RESULTS}

As of fall 2010, Alley and Albuquerque of this study have fitted more than 20 patients while perfecting the $\mathrm{TH}$ and TR CRS socket designs. Some of these patients were fitted with several versions of the socket. In all cases where the patient presented with a preexisting socket, the new socket increased ROM, stability, and capability to handle greater loads in the terminal device. Alley and other CRS-trained prosthetists have fitted over $60 \mathrm{TF}$ patients.

Note that this article is only preliminary. As soon as a number of prosthetists are certified in the design and use of the new Alley CRS socket technique, more detailed studies will be required. For each of the three levels, a statistically valid number of comparison fittings need to be studied. For a valid study, each patient should be fitted with two new sockets in random order. Obtaining valid information from old socket-new socket studies would be convenient, but the fact that most patients only present for a new socket when they no longer fit the old one would make such a study of dubious value. However, if research were to document that the reason for changing sockets was due to a desire for greater performance rather than due to a poor fit, this would be of value. One study that is currently being developed will perform a two-arm randomized crossover analysis comparing a newly created traditional ischial containment socket with its CRS counterpart.

\section{CONCLUSION}

True control of the location of the underlying bone of an encapsulated limb has been largely absent since the origin of prosthetic sockets. Many reasons exist as to why this control has been overlooked. They include a limited knowledge of biomechanics and hydrostatic theory as they relate to interface creation, as well as a failure to understand window edema and its relation to aperture design and location. In the past, prosthetists have focused on proximal control as the primary element in socket biomechanics.

CRS sockets introduce an entirely new approach to interface design that focuses primarily on controlling the location of the underlying bone with respect to the socket or interface walls. This control is applied along the entire shaft of the bone by radial forces that are generated by a series of compression and release areas. With this approach, far greater control of the underlying bone is achieved. The 
benefits of gaining firm control of the encapsulated bone are numerous and far-reaching, extending into all levels of prosthetics and orthotics. We hope that the allied health community will begin to appreciate the role of the prosthetic/orthotic interface beyond just simple comfort, fit, and general function. Our focus should expand its scope to encompass techniques that maximize the wearers' performance. CRS sockets appear to offer superior biomechanical attributes to achieve this expansion.

Sufficient patients have been fitted at each of the three levels of amputation discussed to prove that these new interfaces offer enhanced performance regarding stability, comfort, energy efficiency, ROM, and the perceived weight of the prosthesis by providing a better, more intimate fit that allows increased functionality. We caution readers, however, that further research is needed to quantify how much pressure can be tolerated where the socket approaches the underlying bones. Consequently, more work is required before the sockets can be safely and routinely applied. We are working to create a standard protocol so prosthetists can be trained to use the new design routinely in their practices through a certification program based on this research.

\section{ACKNOWLEDGMENTS}

\author{
Author Contributions: \\ Development of CRS socket design and writing of text: R. D. Alley. \\ Theoretical analysis of CRS concept and writing of text: \\ T. W. Williams III. \\ Development of patient fitting techniques: R. D. Alley, M. J. \\ Albuquerque. \\ Quantification of tissue pressure safety and perfusion analysis: \\ D. E. Altobelli.
}

Financial Disclosures: R. D. Alley and T. W. Williams III have applied for a patent on the design of the socket and the fitting jig described in this article. Their businesses may benefit from the sale of the jig. Alley may additionally benefit from certification courses and/ or the licensing of the CRS technology.

Funding/Support: Some of this material is the result of work supported with resources and the use of facilities at the DEKA Research and Development Corp, Manchester, New Hampshire, under contract with the Defense Advanced Research Projects Agency (DARPA) in the Revolutionizing Prosthetics Project initiative.

Additional Contributions: We wish to acknowledge and thank the patient participants and their family members; DEKA Research and Development, especially Chuck Hildreth, who has been fitted with numerous sockets during the development of the new system; Stewart Coulter and Wayne Penn, DARPA; COL Geoffrey Ling; and the Department of Veterans Affairs. We extend special thanks to Matthew Garibaldi, CPO, currently at the University of California, San Fran- cisco, who was instrumental in applying the CRS technique to TF design as well as providing clinical data from ongoing studies. Additional mention of prosthetic technicians/clinicians is warranted regarding the development of the fabrication techniques for CRS sockets. D. Beauchemin and D. Newman of Next Step and Jose Ceja of New Dimension Prosthetic Custom Fabrication, Los Angles, California, worked on the TH and TR sockets.

Institutional Review: The New England Institutional Review Board reviewed and approved the participation and treatment of Chuck Hildreth, a subject in the DEKA prosthetic arm clinical study and a patient of M. J. Albuquerque.

Participant Follow-Up: The authors plan to inform participants of the publication of this study.

Disclaimer: The intention of this article is to provide a brief overview of the basic theory behind the patent-pending CRS socket/interface system. It is in no way intended to encourage and/or teach clinicians not certified in its proper design and implementation to create and/or attempt to use and fit the CRS technology.

\section{REFERENCES}

1. Gorton A. The "Muenster-type" below-elbow prosthesis: A field study. Inter-Clin Inform Bull. 1966;6(1):12-18.

2. Billock JN. The Northwestern University supracondylar suspension technique for below-elbow amputations. Orthot Prosthet. 1972;26(4):16-23.

3. Fishman S, Kay HW. The Muenster-type below-elbow socket: An evaluation. Artif Limbs. 1964;10:4-14.

[PMID: 14274148]

4. McLaurin CA, Sauter WF, Dolan CM, Hartmann GR. Fabrication procedures for the open-shoulder above-elbow socket. Artif Limbs. 1969;13(2):46-54. [PMID: 5380879]

5. Andrew JT. Elbow disarticulation and transhumeral amputation: Prosthetic principles. In: Bowker JH, Michael JW, editors. Atlas of limb prosthetics: Surgical, prosthetic, and rehabilitation principles. 2nd ed. St. Louis (MO): Mosby Year Book; 1992.

6. Andrew JT. Transhumeral and elbow disarticulation anatomically contoured socket considerations. J Prosthet Orthot. 2008;20(3):107-17. DOI:10.1097/JPO.0b013e31817ded98

7. Lake C. The evolution of upper limb socket design. J Prosthet Orthot. 2008;20(3):85-92.

DOI:10.1097/JPO.0b013e31817d2f08

8. Alley RD. Advancement of upper extremity prosthetic interface and frame design. Proceedings UNB Myoelectric Controls/Powered Prosthetics Symposium; 2002; Fredericton, Canada. Fredericton (Canada): University of New Brunswick: 2002. p. 28-32.

9. Alley RD, Sears HH. Powered upper limb prosthetics in adults. In: Mazumdar M, editor. Powered upper limb prostheses: Control, implementation and clinical application. Berlin (Germany): Springer-Verlag; 2004. p. 134-37. 
10. Murphy EF. Transferring load to flesh-Part I. Concepts. Bull Prosthet Res. 1971;10(16):38-44.

11. Bennett L. Transferring load to flesh-Part II. Analysis of compressive stress. Bull Prosthet Res. 1971;10(16):45-63.

12. Gottschalk F. Transfemoral amputation: Surgical management. In: Smith DG, Michael JW, Bowker JH, editors. 3rd ed. Atlas of amputations and limb deficiencies: Surgical, prosthetic, and rehabilitation principles. Rosemont (IL): American Academy of Orthopaedic Surgeons; 2004. p. 533-40.

13. Schuch CM, Pritham CH. Transfemoral amputation: Prosthetic management. In: Smith DG, Michael JW, Bowker $\mathrm{JH}$, editors. 3rd ed. Atlas of amputations and limb deficiencies: Surgical, prosthetic, and rehabilitation principles. Rosemont (IL): American Academy of Orthopaedic Surgeons; 2004. p. 547-51.

14. Miguelez JM, Lake C, Conyers D, Zenie J. The Transradial Anatomically Contoured (TRAC) Interface: Design principles and methodology. J Prosthet Orthot. 2003;15(4):148-56.

15. Plettenburg DH. Upper extremity prosthetics: Current status and evaluation. Delft (the Netherlands): VSSD; 2006.

16. Plettenburg DH. Basic requirements for upper extremity prostheses: The WILMER approach. Proceedings of the 20th Annual International Conference of the IEEE Engineering in Medicine and Biology Society; 1998 Oct 29-Nov 1; Hong Kong, China. Los Alamitos (CA): IEEE; 1998.
17. Sauter WF, Naumann S, Milner M. A three-quarter type below-elbow socket for myoelectric prostheses. Prosthet Orthot Int. 1986;10(2):79-82. [PMID: 3774515]

18. Daly WK. Electrodes installed in roll-on suspension sleeves. Proceedings UNB Myoelectric Controls/Powered Prosthetics Symposium; 2002; Fredericton, Canada. Fredericton (Canada): University of New Brunswick (Fredericton): 2002. p. 45-47.

Submitted for publication December 14, 2009. Accepted in revised form November 29, 2010.

This article and any supplementary material should be cited as follows:

Alley RD, Williams TW III, Albuquerque MJ, Altobelli DE. Prosthetic sockets stabilized by alternating areas of tissue compression and release. J Rehabil Res Dev. 2011; 48(6):679-96. DOI:10.1682/JRRD.2009.12.0197

ResearcherID: Randall D. Alley, CP, LP: C-8095-2011

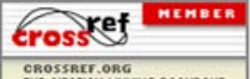

